that interfere with formation of viral capsids. As such, the structure reveals the details of an allosteric site in CA that can be targeted by small-molecule inhibitors.

These studies identify the first inhibitor directed against the formation of immature as well as mature HIV-1 particles, and could be developed for antiviral treatment. Unfortunately, CAI itself cannot be used therapeutically; as with many peptides, the half-life is short and the bioavailability is poor. However, the peptide does represent a suitable starting point for drug development.

Melanie Brazil

6) References and links ORIGINAL RESEARCH PAPERS Sticht, J. et al. A peptide inhibitor of $\mathrm{HIV}-1$ assembly in vitro. Nature Struct. Mol. Biol. 12, 671-677 (2005) | Ternois, F. et al. The HIV-1 capsid protein C-terminal domain in complex with a virus assembly inhibitor. Nature Struct. Mol. Biol. 12, 678-682 (2005) FURTHER READING Pommier, Y., Johnson, A. A. \& Marchand, C. Integrase inhibitors to treat HIV/ AIDS. Nature Rev. Drug Discov. 4, 236-248 (2005) Matthews, T. et al. Enfuvirtide: the first therapy to inhibit the entry of HIV-1 into host CD4 lymphocytes. Nature Rev. Drug Discov. 3, 215-225 (2004) I Stone, A. Microbicides: a new approach to preventing HIV and other sexually transmitted infections. Nature Rev. Drug Discov. 1, 977-985 (2002)

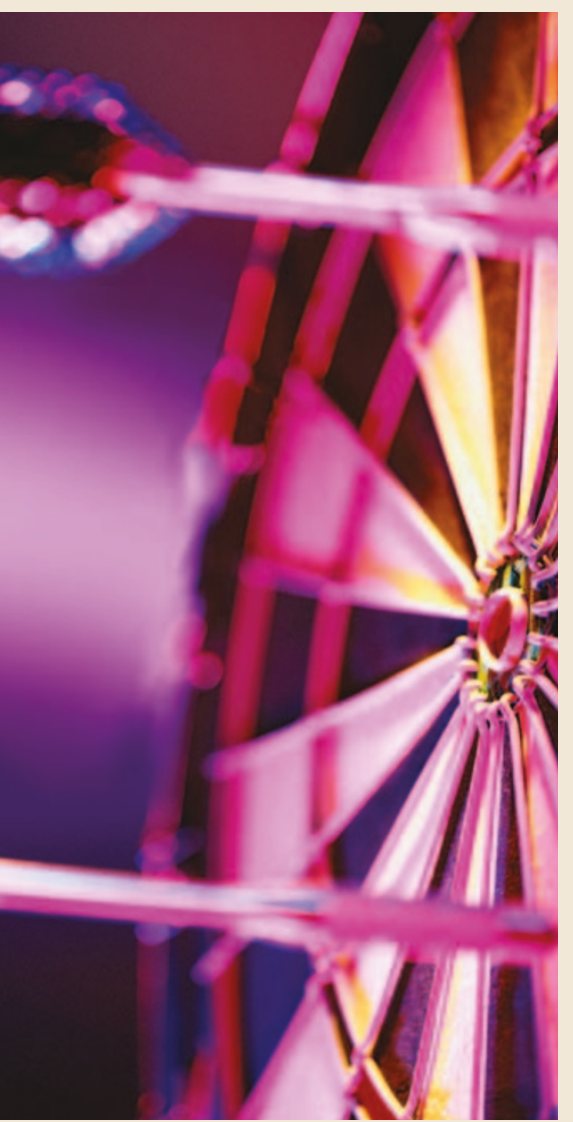

\section{Knocked down for the count}

After the initial excitement surrounding the use of RNA interference (RNAi) as a potential therapeutic modality, there have been substantial hurdles to developing RNAi-based drug candidates. Because unmodified short interfering RNAs (siRNAs) are highly unstable in vivo, the first hurdle was the need to create therapeutically viable siRNAs with improved stability, potency and duration of effect in humans. At least one chemically modified siRNA is in human clinical trials, which demonstrates the success of industry efforts in producing stable, effective therapeutic agents. There remains, however, another hurdle to the widespread application of siRNA to human disease: systemic delivery, and particularly the need to reduce the large doses of siRNAs currently required to achieve prolonged mRNA knockdown. But progress is finally being made on this front. In a recent issue of Nature Biotechnology, David Morrissey and colleagues describe how a stable nucleic-acid-lipid particle (SNALP) containing chemically stabilized siRNA causes persistent knockdown of hepatitis $B$ virus (HBV) replication genes in mice.

Morrissey and colleagues had previously reported that stabilizing siRNAs by substituting all $2^{\prime}-\mathrm{OH}$ residues on the $\mathrm{RNA}$ with $2^{\prime} \mathrm{F}, 2^{\prime} \mathrm{O}-\mathrm{Me}$ or $2^{\prime} \mathrm{H}$ residues dramatically improves the in vivo stability of siRNA. However, the dosing regimen used to demonstrate this in a mouse model of $\mathrm{HBV}$ replication was not therapeutically viable because it required administration of large amounts of siRNA three times daily. By encapsulating the stabilized siRNA in a specialized liposome, the authors were able to use a lower dose while improving the potency and duration of siRNA activity.

SNALPs consist of a lipid bilayer comprising cationic and fusogenic lipids, which enable cellular uptake and endosomal release of the siRNA, coated with a polyethylene glycol (PEG)-lipid conjugate that shields and stabilizes the particle, thereby preventing rapid clearance. When intravenously injected, the PEG-lipid conjugate dissociates from the SNALP particle, which can then go on to transfect cells in vivo.

Initial biodistribution and pharmacokinetic studies showed that substantial levels of SNALP accumulated in the liver and spleen of injected mice and that the siRNA-SNALP had a significantly longer half-life in the liver than the stabilized siRNA particle alone. In mice

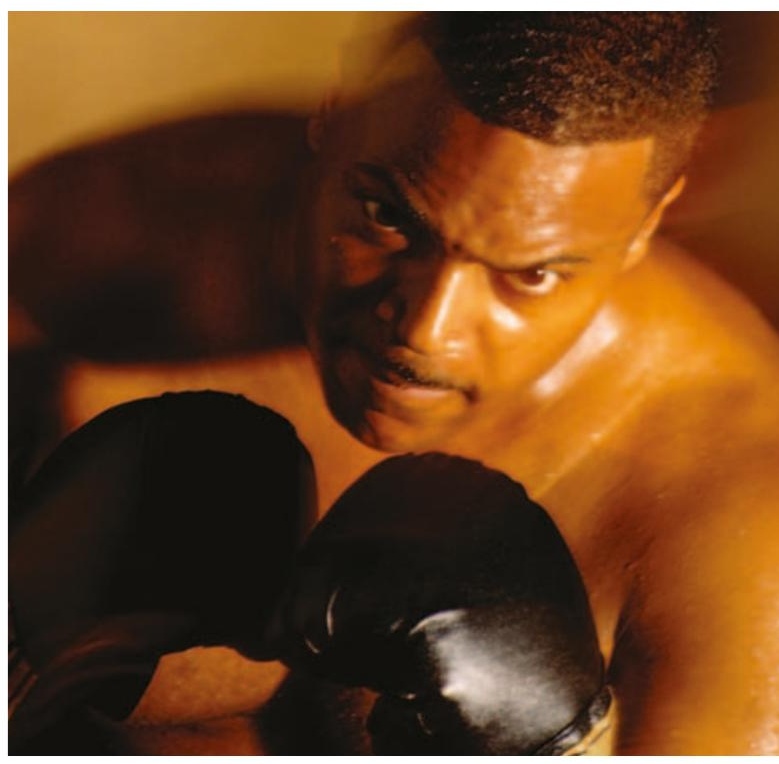

undergoing chronic treatment with SNALP, these pharmacokinetics were maintained throughout a 5-week treatment protocol.

The authors then tested the efficacy of siRNASNALP in a mouse model of HBV replication. Substantial dose-dependent reductions in serum HBV DNA levels were observed 7 days after administration of anti-HBV siRNA-SNALP and, importantly, similar reductions in $\mathrm{HBV}$ replication were maintained over a 6-week course of treatment. A more potent and persistent siRNA-mediated gene-silencing effect was achieved using lower and fewer doses than has been reported previously in the literature.

One well-documented issue with RNAi-based therapeutics is the induction of type 1 interferon inflammatory cytokine immune responses in mammalian cells. Morrissey and colleagues tested the potential for chemically modified siRNA-SNALPs to stimulate such a response, and found that, unlike the unmodified siRNA alone, the chemically modified siRNAs used in the SNALP formulation did not induce interferon- $\alpha$ and inflammatory cytokines in the serum of injected mice.

The authors speculate that the SNALP formulation could minimize the chances of developing oligonucleotide-related toxicities associated with antisense therapies, which have generally been related to dose. Their results are an important step towards making RNAi-based therapeutics a reality.

Joanna Owens

(9) References and links ORIGINAL RESEARCH PAPER

Morrissey, D. V. et al. Potent and persistent in vivo activity of chemically modified siRNAs. Nature Biotech. 23, 1002-1007 (2005) FURTHER READING Morrissey, D. V. et al. Activity of a stabilized short interfering RNA in a mouse model of hepatitis B virus replication. Hepatology 41, 1349-1356 (2005) | Dorsett, Y. \& Tuschl, T. siRNAs: applications in functional genomics and potential as therapeutics. Nature Rev. Drug Discov. 3, 318-329 (2004) 